\title{
Antibiotic Resistance Pattern of Escherichia coli and Klebsiella Species in Pakistan: A Brief Overview
}

\section{Arif Mand Sohail Afzal $\mathbf{M}^{*}$}

School of Science, University of Management and Technology (UMT), Lahore, Pakistan

\begin{abstract}
Antibiotics kill or inhibit the replication of bacteria by different means, like emergence of resistance to these antibiotics is serious threat to health care system across the globe. Pathogens can attain drug resistance either by intrinsic mechanism or may be acquired due to selective pressure of a drug. Escherichia coli (E. coli) and Klebsiella specie (K. species) are the most common causative pathogens for most of the infections especially in countries with poor health care systems. Increase in extended-spectrum $\beta$-lactamases (ESBL) production in these microbes in recent years has led to limitations of treatment. Pakistan is also one of these countries with very low budget for health and per capta income. In Pakistan, majority of health care professionals prescribe antibiotic without testing the pathogen for antibiotic susceptibility. Long term utilization of antibiotics has contributed to greater resistance among pathogenic bacteria. The prevalence of such organisms has brought new challenges for the practitioners treating bacterial infections. The recent studies on the antibiotic resistance pattern of $E$. coli and K. species from Pakistan are summarized and data is showing that the emergence and rapid spread of multidrug resistance in these microbes are of great concern for future. There is an urgent need of community education for health care providers and general population regarding careful utilization of antibiotics.
\end{abstract}

Keywords: Antibiotic; Resistance; E. coli; K. species

\section{Methods and Materials}

Penicillin was the first natural antibiotic that was discovered by Alexander Flaming. First, it was obtained from fungus (Rhizopus) and later on extracted from other fungal strains and from bacteria. Nowadays, antibiotics are synthesized in labs and commercial scale for different applications. Antibiotics have the ability to kill or inhibit the division and multiplication of the microbes [1]. There are number of ways how an antibiotic attacks a germ, like it retards bacterial growth by ceasing DNA replication, stopping RNA synthesis, denaturing important protein or inhibiting protein synthesis, denaturing cell wall and by inhibiting the tetra-hydrofolate synthesis [2].

\section{Antibiotic resistance}

Antibiotic resistance means that drug susceptible response is lost against microbial an infection which has become an emerging problem globally. This resistant behavior of microbes against drugs could be due to intrinsic or acquired responses against drugs. As it is a global problem there is need to conduct research on different microbes and their antibiotic resistance behavior [3,4]. Antibiotic can be broadly classified.

\section{Intrinsic resistance}

It is an adaptive approach in which a microbe tries to resist an antibiotic to sustain survival [5]. It was found that Pseudomonas aeruginosa has a less permeable membrane that are responsible for its multi-drug resistance (MDR), similar structural advantages are found in other bacterial strains as well. As these characteristics are found in wild type strains so this type of resistance is called intrinsic resistance [6]. Beta lactamases is an enzyme that destroys the harmful effect of the anti-microbial agent. K. species and $E$. coli have genes for coding this enzyme and hence exhibit intrinsic resistance [7]. In some bacterial strains natural pumps exist that efflux the toxins from cell wall and hence make it impermeable for the drug [8]. Another method is diverting the metabolic pathways, it will change the target and hence antibiotic could not kill the bacteria [9].

\section{Acquired resistance}

Selective pressure of a drug transforms the natural susceptible fauna into resistant microbes. This kind of immunity is acquired under the need of natural selection and survival of fittest [1]. The excessive use of antibiotics is introducing a selective pressure which is becoming responsible of resistance or even multi-resistance characteristics in some of the bacterial populations [10]. Mutation in genes that control metabolic pathways also results in antibiotic resistance [6]. Integrin genes move from one genetic location to another within the same cell, whereas transposons is the transfer of genes through plasmids via bacteriophages.

\section{Antibiotic resistance in $E$. coli and K. species}

Antibiotic resistance in microbes is a major health concern all over the world and in particular in third world countries. Due to poor waste and water management humans are in continuous contact with microbes and disease causing bacteria. It is found that in hospitals plasmid directed mutations are very high and so antibiotic resistance in E. coli and K. species is also very high. Previously antibiotics resistance of E. coli was tested and found nearly $70 \%$ against streptomycinsulfisoxazole-tetracycline. It was also shown that ampicillin, kanamycin, sulfisoxazole, streptomycin, tetracycline, and ticarcillin have decline in susceptibility [11]. In a study conducted at Nigeria an increasing trend against tetracycline, sulfonamide, streptomycin and ampicillin was found in urine samples [12].

*Corresponding author: Sohail Afzal M, School of Science, University of Management and Technology (UMT), Lahore, Pakistan, Tel: 923215244808; E-mail: sohail.ncvi@gmail.com

Received October 23, 2017; Accepted November 08, 2017; Published November 15, 2017

Citation: Afzal MAMS (2017) Antibiotic Resistance Pattern of Escherichia coli and Klebsiella Species in Pakistan: A Brief Overview. J Microb Biochem Technol 9: 277279. doi: $10.4172 / 1948-5948.1000377$

Copyright: $\odot 2017$ Afzal MAMS. This is an open-access article distributed under the terms of the Creative Commons Attribution License, which permits unrestricted use, distribution, and reproduction in any medium, provided the original author and source are credited. 


\section{Antibiotic resistance pattern in Pakistan}

Antibiotic resistance is increasing in all regions of the world but in developing countries this rate is alarming primarily due to over-dosage of antibiotics. This over exposure to drug is making microbes stronger. In developing countries antibiotics are generally given for very long time and without proper culturing [13]. Pakistan being a developing country is also facing the same problem. Cephalosporin was one of the most frequently prescribed antibiotics and an increase in its resistance has been reported, mainly due to over exposure to drug [14,15].

Pakistan has very high rate of the mortality and morbidity due to nosocomial infection primarily because of over-crowded hospitals with insufficient cleaning and disinfection [14-16]. Different studies across the world have proved that same antibiotic has different resistance or susceptibility pattern for different sites of infection. Most commonly used antibiotic for urinary tract infection (UTI) are amoxicillin+clavulanate, imipenam, piperacillin+tazobactum, and amikacin, etc. [17]. Even among these antibiotics the resistance and susceptibility patterns varies. E. coli and K. species are major causes of many hospitals born and community based diseases in Pakistan. Recently, in a study 14 antibiotics were tested against E. coli and K. species and it was found that drug resistance was increasing in both species. Most of the pathogens isolated from UTI infections showed more than $70 \%$ antibiotic resistance against ceftriaxone, ciprofloxacin, cefixime (unpublished data). E. coli is mainly involved in gastric diseases due to poor quality of drinking water. Previously it has been shown that nearly $50 \%$ strains of E. coli are multi drug resistant (MDR). This is intrinsic as well as acquired resistance. It was found that nearly $76 \%$ strain of $E$. coli showed resistance to ampicillin and resistance also increases against trimethoprime. Ciprofloxacin, ofloxacin and norfioxacin although have shown susceptibility against $E$. coli but still it was $47-50 \%$ [18]. Susceptibility rate of $E$. coli isolated from UTI showed that highest resistance was against ceftriaxone $(82.5 \%)$, followed by nalidixic acid $(23.8 \%)$, cefaclor $(23.1 \%)$, cephradine $(18.9 \%)$ and amoxicillin.

\section{Discussion}

Moreover, it was previously shown that majority of the E. coli, isolates from UTI (73\%), had resistance towards the co-amoxiclav and other, $42 \%$ isolates, to fluoroquinolones $[19,20]$. The recent reports analyzed the antibiotic resistance pattern of $E$. coli which indicates that the most of the isolates exhibit MDR to ampicillin, streptomycin, tetracycline, trimethoprim sulfamethoxazole, nalidixic acid, ciprofloxacin and chloramphenicol $[21,22]$. It was also highlighted recently that emergence of MDR bacteria are on raise and $77.5 \%$ of all the screened isolates were resistant to three or more than three of the tested antibiotics [23]. A report from Sindh province of Pakistan showed that nearly half of the isolates from UTI have shown resistance to most of the commonly used antibiotics recommended in the guidelines especially the floxacin group probably because of its unwarranted use [24]. A large scale retrospective study from Karachi analyzed the data of 27,852 blood cultures over the period of four years $(2010,2012,2014$ and 2015) and the results showed that Klebsiella spp. infection increased over the years from 19 to $35 \%$. The antibiotic resistance pattern may also be changed due to its overexposure e.g. imipenem resistance was lowest in 2010 around $1 \%$ which has increased to $20 \%$ in 2015 [25]. Antibiotic resistance in $\mathrm{K}$. species in Khyber Pakhtunkhwa is also quite high, $71 \%$ isolates were resistant to 3 or more antibiotics (Multidrug resistant). Resistance to co-trimoxazole was highest followed by gentamicin, doxycycline, enoxacin, ciprofloxacin, gatifloxacin and kanamycin [26]. However, it was also shown that $\mathrm{K}$. species have sensitivity to ceftazidime and ciprofloxacin above $95 \%$. Other studies showed sensitivity of K. species to cefotaxime above $70 \%$, ceftriaxone above $75 \%$ and ciprofloxacin above $63 \%$ in case of UTI $[14,15]$.

In Pakistan low literacy rate and corruption in law enforcement agencies allows illegal practitioners to give over dose of medicines without any screening tests. They generally prescribe third degree broad spectrum antibiotics which pave way for antibiotic resistance. In last two decades poultry and cattle farming has increased in Pakistan and mostly antibiotics are given to these animals in the form of water solutions. There is no separate drainage system for this industry and untreated water is released in main sewage system. This cause contamination of ground water table with antibiotics and this continuous exposure to drugs make it microbes more resistant.

\section{Conclusion}

E. coli and K. species are the major cause for most of infections in Pakistan. There is lacking evidence about the growing antibiotic resistance and molecular pathways involved in this resistance in these pathogens due to very limited available data in Pakistan. Most of the recent research on this topic is from Western countries where climatic, social and environmental conditions are quite different from developing countries with a distinct host-pathogen interaction. These differences are leading to empirical therapy which is based on the local susceptibility profiles; therefore a revised line of management should be developed locally in accordance with the susceptibility pattern of the pathogens to avoid further resistance as well as morbidity of the patient. Therefore it is recommended that large scale studies analyzing the antibiotic resistance pattern in infectious bacteria should be carried out in the country on regular basis. This will help in designing the anti-bacteria therapy according to the local needs. Hence, there is an urgent need of improvement in the health care system of Pakistan. The increasing trend of antibiotic resistance due to over dosage of drug can be reduced by educating the health care providers. It is recommended that Government and Non-Government organization should take part in awareness campaigns not only for general community but also for medical practitioners.

\section{References}

1. Ahmad S, Aljuaid FN, Alenzi QF, Matter HE, Bakheet EO (2009) Prevalence antibiotic susceptibility pattern and production of extended spectrum beta lactamases amongst clinical isolates of Klebsiella pneumoniae at armed forces hospital in Saudi Arabia. J Coll Physicians Surg Pak 19: 264-265.

2. Finch RG (2004) Antibiotic resistance: A view from the prescriber. Nat Rev Microbiol 2: 989-994.

3. Mehrgan H, Rahbar M, Arab-Halvaii Z (2009) High prevalence of extendedspectrum beta-lactamase-producing Klebsiella pneumoniae in a tertiary care hospital in Tehran, Iran. J Infect Dev Ctries 4: 132-138.

4. Kumari HBV, Nagarathna S, Chandramuki A (2007) Antimicrobial resistance pattern among aerobic gram negative bacilli of lower respiratory tract specimens of intensive care unit patients in a neurocentre. Indian J Chest Dis Allied Sci 86: 408-416.

5. Normark BH, Normark S (2002) Evolution and spread of antibiotic resistance. J Intern Med 252: 91-106.

6. Yoneyama $\mathrm{H}$, Katsumata $\mathrm{R}$ (2006) Antibiotic resistance in bacteria and its future for novel antibiotic development. Biosci Biotechnol Biochem 70: 1060-1075.

7. Abraham EP, Chain E (1940) An enzyme from bacteria able to destroy penicillin Nat Rev Microbiol 146: 837

8. Levy SB, McMurry L (1974) Detection of an inducible membrane protein associated with $\mathrm{R}$-factor-mediated tetracycline resistance. Biochem Biophys Res Commun 56: 1060-1068. 
9. Amyes SGB, Smith JT (1974) R-factor trimethoprim resistance mechanism: an insusceptible target site. Biochem Biophys Res Commun 58: 412-418.

10. Chen Z, Jiang X (2014) Microbiological safety of chicken litter or chicken litterbased organic fertilizers: A review. Agri 4: 1-29.

11. Tadesse DA, Zhao S, Tong E, Ayers S, Singh A, et al. (2012) Antimicrobial drug resistance in Escherichia coli from humans and animals, United States, 19502002. Prev Chronic Dis18: 741.

12. Okeke IN, Fayinka ST, Lamikanra A (2000) Antibiotic resistance in Escherichia coli from Nigerian students, 1986-1998. Emerg Infect Dis 6: 393.

13. Grover SS, Sharma M, Pasha ST, Singh G, Lal S (2004) Antimicrobial susceptibility pattern and prevalence of extended spectrum beta lactamases producing strains of Klebsiella pneumonia from a major hospital in New Delhi. J Commun Dis 36: 17-26.

14. Shaikh JM, Devrajani BR, Shah SZ, Akhund T, Bibi I (2007) Frequency pattern and etiology of nosocomial infection in intensive care unit: An experience at a tertiary care hospital. J Ayub Med Coll Abbottabad 20: 37-40.

15. Sheikh D, Ahmad F, Naqvi SB, Shoaib MA, Hashmi K (2002) Resistance pattern of different aminoglycosidase antibiotics against gram positive and gram negative clinical isolates from, Karachi. Pak J Pharm Sci 15: 57-67.

16. Shahid M, Malik A, Akram M, Agarwal ML, Khan UA, et al. (2008) Prevalent phenotypes and antibiotic resistance in Escherichia coli and Klebsiella pneumonia at an Indian tertiary care hospital: Plasmid-mediated cefoxitin resistance. Int J Infect Dis 2: 256-264.

17. Ahmad W, Jamsheed $F(2015)$ Frequency of $E$. coli in community acquired urinary tract infection and their resistance pattern against common antibacterials. J Ayub Med Coll Abbottabad 27: 333-337.

18. Iqbal M, Patel IK, Ain Q, Rabani KZ, Shah SH (2002) Susceptibility pattern of
E. coli: Prevelance of multidrug-resistant isolates and extended spectrum BetaLactamase phenotype. J Pak Med Assoc 52: 47

19. Ali I, Kumar N, Ahmed S, Dasti Jl (2014) Antibiotic resistance in uropathogenic E. coli strains isolated from non-hospitalized patients in Pakistan. J Clin Diagn Res 8: DC01-4.

20. Tanvir R, Hafeez R, Hasnain S (2012) Prevalence of multiple drug resistant $E$. coli in patients of Urinary tract infection registering at a diagnostic laboratory in Lahore. Pak J Zool 44: 707-712.

21. Bashir S, Haque A, Sarwar Y and Raza A (2015) Prevalence of integrons and antibiotic resistance among uropathogenic Escherichia coli from Faisalabad region of Pakistan. Arch Clinic Microb 6: 4-9.

22. Sohail M, Khurshid M, Saleem HG, Javed H, Khan AA (2015) Characteristics and antibiotic resistance of urinary tract pathogens isolated from Punjab, Pakistan. Jundishapur J Microbiol: 8.

23. Bashir S, Sarwar Y, Ali A, Mohsin M, Saeed MA, et al. (2011) Multiple drug resistance patterns in various phylogenetic groups of uropathogenic $E$. coli. Braz J Microbiol 42: 1278-1283.

24. Kidwai SS, Nageen A, Ghaznavi S, Bashir F, Ara J (2017) Antibiotic susceptibility in commonly isolated pathogens from urinary tract infection in a cohort of subjects from low socioeconomic strata. Pak J Med Sci 33: 254-259.

25. Dodani S, Nasim A (2017) Changing trends in antimicrobial resistance pattern in Klebsiella species bacteremia in an out-patient hemodialysis unit over 6 years (2010-2015) in a tertiary care hospital in Pakistan. Open Forum Infect Dis 4: S156.

26. Ullah F, Malik SA, Ahmed J (2009) Antimicrobial susceptibility pattern and ESBL prevalence in Klebsiella pneumoniae from urinary tract infections in the NorthWest of Pakistan. Afr J Microbiol Res 3: 676-680.
Citation: Afzal MAMS (2017) Antibiotic Resistance Pattern of Escherichia coli and Klebsiella Species in Pakistan: A Brief Overview. J Microb Biochem Technol 9: 277-279. doi: 10.4172/1948-5948.1000377 